\title{
Singlet-Assisted Supersymmetry Breaking
}

\author{
Yael Shadmi ${ }^{1}$ and Yuri Shirman ${ }^{2}$ \\ ${ }^{1}$ Physics Department, Technion-Israel Institute of Technology, \\ Haifa 32000, Israel \\ ${ }^{2}$ Department of Physics and Astronomy, \\ University of California, Irvine, CA 92697, USA
}

(Dated: October 2011)

\begin{abstract}
We describe a simple recipe for obtaining local supersymmetry-breaking vacua in s-confining theories coupled to gauge singlets. This recipe gives rise to effective O'Raifeartaigh models in the IR, with calculable supersymmetry-breaking minima near the origin, and can be applied to both vector-like and chiral theories. Since the properties of the vacuum are largely determined by superpotential terms that are non-renormalizable in the UV, it is calculable even when all dimensionless couplings are taken to be of order one. By construction, the models preserve a large subgroup of the original global symmetry. While we only study here s-confining theories, we expect our tools to be useful for inducing dynamical supersymmetry breaking in many gauge theories.
\end{abstract}

PACS numbers: 12.60.Jv,11.30.Hv 


\section{INTRODUCTION}

Dynamical supersymmetry breaking (DSB) provides a beautiful mechanism for generating the hierarchy between the weak scale and the Planck scale [1]. One of the least satisfying aspects of this idea is the lack of a general organizing principle for finding DSB theories [2]. This is related to the fact that these theories are non-generic. It is reasonable to expect this problem to be ameliorated if one does not insist on global supersymmetry-breaking minima, and instead only searches for local minima. Indeed, local DSB minima were found to occur in some of the simplest supersymmetric gauge theories, namely supersymmetric QCD [3], suggesting that local DSB vacua are quite generic. Furthermore, if one only requires a local minimum, it may not be necessary to lift all of the classical flat directions, so that the classical superpotential one adds can be less intricate, and the resulting models more generic.

Simpler superporpotentials have another potential advantage. The classical superpotential necessarily breaks some of the global symmetry of the original theory. This is problematic for constructing models of direct gauge mediation, since these are obtained by embedding the standard model gauge group in a (weakly gauged) subgroup of the global symmetry of the DSB model [4]. If one is not forced to lift all the classical flat directions, a larger subgroup of the original global symmetry is likely to survive.

In this paper, we describe a simple recipe for obtaining DSB minima in many s-confining theories [5 8], by coupling these theories to gauge singlets. Essentially, the construction yields an effective O'Raifeartaigh model in the IR, with a supersymmetry breaking vacuum near the origin of the moduli space, much like in the models of [3]. Our recipe can be applied to both vector-like theories, such as s-confining SQCD, and to chiral theories (for metastable susy breaking in chiral theories without singlets see [9, 10]). It is also closely related to the models of [11, 12] in which DSB is achieved through couplings to gauge singlet fields.

In order to obtain the supersymmetry-breaking minimum near the origin, we do not need to lift all of the classical flat directions. Just as in [11, 12], the classical moduli space is parametrized by the gauge singlets. The fate of this classical moduli space is somewhat model dependent. As we will see, in some of the s-confining theories it is lifted non-perturbatively like in the models of [11, 12], while in others, for large field VEVs, there are runaway directions along which the potential asymptotes to zero. In any case, the supersymmetry-breaking vacuum near the origin is separated from these by a non-calculable potential barrier.

Specifically, we will introduce singlet fields $S_{I}$ for each of the gauge invariants $O_{I}$ of the s-confining theory, apart from a single one. Denoting this last gauge invariant by $O_{0}$, we add the superpotential ${ }^{1}$,

$$
W=S_{I} O_{I}+O_{0}
$$

Below the confinement scale of the theory, the last term of Eq. (11) becomes a tadpole. Since the non-perturbative dynamics of s-confining theories often gives rise to superpotential terms that are cubic in the gauge invariants, the IR theory reduces to an O'Raifeartaigh model. In some cases, such as s-confining SQCD, all the IR fields appear in the O'Raifeartaigh

\footnotetext{
${ }^{1}$ The addition of a tadpole for a gauge invariant modulus is required to exclude the supersymmetric minimum at the origin of the field space. In the models of [11, 12] such a tadpole was not necessary since the origin did not belong to the quantum moduli space.
} 
superpotential. In more complicated examples, the gauge invariants separate into two sets. One set constitutes an O'Raifeartaigh model, while the second set consists of massive fields whose only superpotential interactions are non-renormalizable (NR). Since these latter fields obtain masses in the IR through the superpotential Eq. (1), their vacuum expectation values $(\mathrm{VEVs})$ vanish and they do not affect the supersymmetry-breaking minimum.

Most of the gauge invariants $O_{I}$ have dimensions larger than 2. The coefficients of the corresponding terms in Eq. (11) are then naturally small. In particular, choosing $O_{0}$ to be the highest dimension gauge invariant results in a calculable minimum, without having to take any order-one parameters to be small.

We only study s-confining theories in this paper. These are particularly simple to analyze since their moduli spaces are parametrized by the gauge invariants $O_{I}[5]$. Furthermore, all of these theories have been found and their superpotentials are known in [5, 8, 13]. However, we expect this construction to be applicable in a wide variety of models, including models with IR-free dual descriptions.

The construction described above gives rise to the simplest O'Raifeartaigh models in the IR, and therefore leaves the $R$-symmetry unbroken. As we will show, however, slight variations of these models give O'Raifeartaigh-type models with some fields having $R$-charges different from 0 or 2 as in [14], leading to $R$-breaking minima. This requires taking some couplings that would naturally be order one, to be small.

As mentioned above, one of the inherent difficulties in using DSB models in the context of direct gauge mediation is that the superpotential required for lifting the flat directions breaks some of the global symmetry. The recipe we described above is clearly advantageous from this point of view, since the $S_{I} O_{I}$ terms do not break any symmetries. In fact, the $S_{I}$ 's and $O_{I}$ 's are in conjugate representations of the global symmetry, and can therefore be used as vector-like messenger pairs once the standard-model subgroup of that global symmetry is gauged.

This paper is organized as follows. In Sec. II, we study in detail the even- $n \mathrm{SU}(n)$ theories with a single anti-symmetric tensor and four flavors. We will show that these have a calculable supersymmetry-breaking minimum near the origin, and no supersymmetric minima for VEVs smaller than the cutoff scale. We will also present an $R$-symmetry breaking modification of the basic model. We generalize this construction to the even- $n$ theories in Sec. II. The remaining s-confining $\mathrm{SU}(n)$ theories are discussed in Sec. IV].

\section{THE EVEN- $n$ SINGLE ANTI-SYMMETRIC TENSOR SU $(n)$ THEORIES}

As a first example, we take $\mathrm{SU}(2 N)$, with a single antisymmetric tensor, and four flavors. This is a chiral theory - only four pairs of fundamentals and anti-fundamentals can be given mass. The field content of the theory is summarized in the first part of Table【. This theory was studied in [7, 8]. We have listed in the Table [ the anomaly-free global symmetry of the theory before the addition of any tree-level superpotential.

While this might seem as a complicated first example to choose, we will see that the fields of the IR theory "factorize" into two sets. One set only appears in those terms of the dynamical superpotential that remain non-renormalizable in the IR and is therefore irrelevant for studying the potential near the origin, while the second set constitutes an O'Raifeartaigh model. This factorization is generic to the s-confining theories.

The IR theory can be described in terms of the gauge invariants defined in the second 


\begin{tabular}{|c|c|c|c|c|c|c|c|}
\hline & $S U(2 N)$ & $S U(2 N)$ & $S U(4)$ & $U(1)^{\prime}$ & $U(1)$ & $U(1)_{R}$ & $\operatorname{dim}$ \\
\hline$A$ & $\boxminus$ & 1 & 1 & 0 & 4 & 0 & 1 \\
$\bar{Q}$ & $\bar{\square}$ & $\square$ & 1 & 4 & 0 & $1 / N$ & 1 \\
$Q$ & $\square$ & 1 & $\square$ & $-2 N$ & $-2(N-1)$ & 0 & 1 \\
\hline \hline$M_{i a} \sim\left(Q_{i} \bar{Q}_{a}\right)$ & & $\square$ & $\square$ & $4-2 N$ & $-2(N-1)$ & $1 / N$ & 2 \\
$P_{a b} \sim\left(A \bar{Q}^{2}\right)$ & & $\boxminus$ & 1 & 8 & 2 & $2 / N$ & 3 \\
\hline$Y_{i j} \sim\left(A^{(N-1)} Q^{2}\right)$ & & 1 & $\boxminus$ & $-4 N$ & 0 & 0 & $N+1$ \\
$X \sim\left(A^{N-2} Q^{4}\right)$ & & 1 & 1 & $-8 N$ & $-4 N$ & 0 & $N+2$ \\
$B \sim(\bar{Q})^{2 N}$ & & 1 & 1 & $8 N$ & 0 & 2 & $2 N$ \\
$\tilde{A} \sim\left(A^{N}\right)$ & & 1 & 1 & 0 & $4 N$ & 0 & $N$ \\
\hline \hline$S_{M}^{i a}$ & & $\square$ & $\square$ & $-4+2 N$ & $2(N-1)$ & $2-1 / N$ & 1 \\
$S_{P}^{a b}$ & & $\bar{G}$ & 1 & -8 & -2 & $2-2 / N$ & 1 \\
$S_{Y}^{i j}$ & & 1 & - & $4 N$ & 0 & 2 & 1 \\
$S_{X}$ & & 1 & 1 & $8 N$ & $4 N$ & 2 & 1 \\
$S_{A}$ & & 1 & 1 & 0 & $-4 N$ & 2 & 1 \\
\hline
\end{tabular}

TABLE I: Matter content of the $S U(2 N)$ model. The quantum numbers for charged elementary fields are given in the top part, for gauge invariant composites in the middle part, and for gauge singlets in the bottom part.

part of Table 【,

$$
M_{i a}, \quad P_{a b}, \quad Y_{i j}, \quad X, \tilde{A}, \quad B,
$$

where $a, b=1, \ldots, 2 N$ and $i, j=1, \ldots, 4$, with the dynamically generated superpotential

$$
W_{d y n}=\frac{1}{\Lambda^{4 N-1}}\left\{\left[\tilde{A} M^{4} P^{N-2}+Y M^{2} P^{N-1}+X P^{N}\right]+\left[B\left(\tilde{A} X+Y^{2}\right)\right]\right\} .
$$

Note that $M$ and $P$ only appear in the first three terms of this superpotential, which are nonrenomalizable and involve high powers of these fields. The last two terms involve $A, X, Y$, and $B$ only, and will become a part of an O'Raifeartaigh model leading to supersymmetry breaking. Here and in the following, we separate the superpotential into two parts, to highlight this factorization

\section{A. Classical superpotential and flat directions}

In order to obtain the basic O'Raifeartaigh model we add singlets for each one of the gauge invariants apart from $B$ (see bottom part of Table I), with the superpotential,

$$
\begin{aligned}
W_{0}= & {\left[\lambda_{M} S_{M}^{i a}\left(Q_{i} \bar{Q}_{a}\right)+\frac{\lambda_{P}}{M_{\mathrm{UV}}} S_{P}^{a b}\left(A \bar{Q}^{2}\right)_{a b}\right]+} \\
& {\left[\frac{\lambda_{A}}{M_{\mathrm{UV}}^{N-2}} S_{A}\left(A^{N}\right)+\frac{\lambda_{Y}}{M_{\mathrm{UV}}^{N-1}} S_{Y}^{i j}\left(A^{(N-1)} Q^{2}\right)_{i j}+\frac{\lambda_{X}}{M_{\mathrm{UV}}^{N}} S_{X}\left(A^{N-2} Q^{4}\right)+\frac{\lambda_{B}}{M_{\mathrm{UV}}^{2 N-3}}(\bar{Q})^{2 N}\right] }
\end{aligned}
$$


where parantheses denote the contraction of $\mathrm{SU}(2 N)$ indices. The $\lambda$ 's are order-one couplings. We could in principle absorb these into the singlets but it would be convenient to keep them explicit for the discussion below. This superpotential lifts all the flat directions associated with the charged fields. The classical moduli space is therefore parameterized by the singlets. In Section IIB we will argue that near the origin of the moduli space all flat directions are lifted and a supersymmetry-breaking local minimum exists. In Section IID we will show that at large VEVs most of the singlet classical flat directions are lifted at the quantum level while the only remaining runaway along the $S_{P}$ direction is separated by a potential barrier from the supersymmetry breaking minimum.

The superpotential Eq. (4) is not the most generic one consistent with the unbroken $\mathrm{SU}(4) \times \mathrm{SU}(2 N) \times \mathrm{U}(1) \times \mathrm{U}(1)_{R}$ global symmetry $\left(\mathrm{U}(1)^{\prime}\right.$ is broken explicitly). However, the only additional tree level terms that can be added to Eq. (4) that are consistent with this symmetry are suppressed compared to terms included in Eq. (41) both in the UV and in the IR. Indeed, new operators are obtained by multiplying the existing terms by $A X$ or by the classically equivalent combination $Y^{2}$. In the UV these operators start at dimension $2 N+5$, whereas the highest dimension term of Eq. (44) has dimension $2 N$. In the IR, these operators start at dimension 4, while the maximal IR dimension of the terms in Eq. (4) is 2.

\section{B. The supersymmetry-breaking vacuum}

We will now show that there is a local supersymmetry-breaking minimum near the origin ${ }^{2}$. For finite field VEVs, the Kähler potential in terms of the gauge invariants is non-singular, so that these gauge invariants can be used to study the IR theory. The last term of Eq. (4) is then linear in the baryon $B$, and the remaining terms become mass terms near the origin. The full superpotential is given by,

$$
\begin{aligned}
W & =\tilde{A} M^{4} P^{N-2}+Y M^{2} P^{N-1}+X P^{N}+B\left(\tilde{A} X+Y^{2}\right) \\
& +m_{M} S_{M}^{i a} M_{i a}+m_{P} S_{P}^{a b} P_{a b}+m_{Y} S^{i j} Y_{i j}+m_{X} S_{X} X+m_{A} S_{A} \tilde{A}+f_{B} B
\end{aligned}
$$

with the masses

$$
m_{M}=\lambda_{M} \Lambda, \quad m_{P}=\lambda_{P} r_{\mathrm{UV}} \Lambda, \quad m_{A}=\lambda_{A} r_{\mathrm{UV}}^{N-2} \Lambda, \quad m_{Y}=\lambda_{Y} r_{\mathrm{UV}}^{N-1} \Lambda, \quad m_{X}=\lambda_{X} r_{\mathrm{UV}}^{N} \Lambda,
$$

and

$$
f_{B}=\lambda_{B} r_{\mathrm{UV}}^{2 N-3} \Lambda^{2}
$$

where we defined

$$
r_{\mathrm{UV}} \equiv \frac{\Lambda}{M_{\mathrm{UV}}} \ll 1
$$

The masses Eq. (6) are hierarchical, with

$$
m_{M} \sim \Lambda \gg m_{P} \gg m_{A} \gg \sqrt{f_{B}} \gg m_{Y} \gg m_{X}
$$

and

$$
m_{x} m_{A} \sim m_{Y}^{2}<f_{B}
$$

$\overline{2}$ This analysis is very similar to the analysis of $[15]$. 
It is easy to see that there are no supersymmetric minima at finite VEVs. The singlet $F$-term equations set all invariants to zero except $B$. The $B$ equation is schematically

$$
A X+Y^{2}+f_{B}=0
$$

and this cannot be satisfied for $A=X=Y=0$.

Near the origin, we can integrate out the heaviest fields, $M, P$ and the corresponding singlets, to get an effective O'Raifeartaigh model with the superpotential,

$$
W=B\left(\tilde{A} X+Y^{2}+f_{B}\right)+m_{Y} S^{i j} Y_{i j}+m_{X} S_{X} X+m_{A} S_{A} A
$$

The behavior of this model is well known. Supersymmetry is broken since the $S_{A}$, $S_{X}$, and $S_{Y}^{i j}$ equations set $A, X$ and $Y_{i j}$ to zero respecively, in conflict with the $B$ equation. At treelevel, there is a flat non-zero potential with $B$ undetermined. This potential is corrected radiatively, and the one-loop Coleman-Weinberg potential leads to a minimum at $B=0$, with small or zero VEVs for the remaining fields. To see this, recall that with no tuning of $\lambda_{A, X, Y}$, we have $m_{A} m_{X} \sim m_{Y}^{2}$. To simplify the problem, we can study the model in two limits. Take first $\lambda_{A}<r_{\mathrm{UV}}$, so that $m_{A}<m_{Y}$. The $Y$ spectrum is then approximately supersymmetric, and we can integrate out $Y$ and $S_{Y}$, recovering the simplest O'Raifeartaigh model, with a minimum at

$$
\begin{aligned}
B & =0, \quad S_{X}=S_{A}=0, \\
A & =\sqrt{\frac{m_{X}}{m_{A}}\left(f_{B}-m_{A} m_{X}\right)}, \quad X=\sqrt{\frac{m_{A}}{m_{X}}\left(f_{B}-m_{A} m_{X}\right)} .
\end{aligned}
$$

Taking instead $\lambda_{Y}<r_{\mathrm{UV}}$ so that $Y$ is lighter than $A, X$, the minimum is at

$$
B=0, \quad S_{Y}=0, \quad Y=\sqrt{f_{B}-m_{Y}^{2}} .
$$

In both of these limits, the $B$ mass is given by

$$
m_{B}^{2(C W)}=\frac{1}{16 \pi^{2}} \frac{m_{1}^{2} m_{2}^{2}}{f_{B}}
$$

with $m_{1} m_{2}=m_{X} m_{A}$ in the former case $\left(\lambda_{A}<r_{\mathrm{UV}}\right)$, and $m_{1} m_{2}=m_{Y}^{2}$ in the latter $\left(\lambda_{Y}<\right.$ $r_{\mathrm{UV}}$ ). Restoring $\lambda_{A} \sim \lambda_{Y} \sim 1$, it is clear then that Eq. (16) still holds, while the VEVs of $A, X$ and $Y$ are somewhere between the values of Eq. (13), Eq. (15) and zero.

All these VEVs are parametrically smaller than $\Lambda$. Using Eq. (66) we can find for the VEVs of $A$ and $X$ of Eq. (13)

$$
\begin{gathered}
A \sim \lambda_{A}^{-1 / 2} r_{\mathrm{UV}}^{N-1 / 2} \Lambda \ll \Lambda \\
X \sim \lambda_{A}^{1 / 2} r_{\mathrm{UV}}^{N-5 / 2} \Lambda \ll \Lambda
\end{gathered}
$$

and for the $Y$ VEV of Eq. (15)

$$
Y \sim r_{\mathrm{UV}}^{N-3 / 2} \Lambda \ll \Lambda
$$




\section{Calculability}

We can now see why the analysis above is reliable. Near the origin, the Kähler potential is roughly of the form

$$
\begin{aligned}
K & =M^{\dagger} M\left[1+g_{M}\left(\frac{M}{\Lambda}, . ., \frac{\lambda_{M} S_{M}}{\Lambda}\right)\right]+B^{\dagger} B\left[1+g_{B}\left(\frac{M}{\Lambda}, . ., \frac{\lambda_{M} S_{M}}{\Lambda}\right)\right] \\
& +S^{\dagger} S\left[1+\lambda_{M} g_{S_{M}}\left(\frac{M}{\Lambda}, . ., \frac{\lambda_{M} S_{M}}{\Lambda}\right)\right]+\ldots
\end{aligned}
$$

where $g_{M}$ etc are non-calculable functions, and the ellipses stand for terms involving the remaining fields.

Since there are mass terms for $M, P, S_{M}, S_{P}$ near the origin, these fields have zero VEVs and zero $F$-terms at the minimum of interest, so the non-calculable Kähler terms involving these fields can be neglected. Likewise, the NR part of the dynamical superpotential involves at least three powers of $M$ and $P$, and therefore does not generate a potential near the origin.

As we saw above, the fields $A, X, Y$ all have vanishing $F$-terms and VEVs much smaller than $\Lambda$, so higher-dimension Kähler terms involving these fields can be neglected. The only potentially dangerous Kähler terms involve $B$, which has a non-zero $F$-term, and therefore generates a potential

$$
V=f_{B}^{2}\left(1+\alpha B^{\dagger} B / \Lambda^{2}+\ldots\right)
$$

where $\alpha$ is a non-calculable order-one coefficient, and the ellipsis stands for similar terms involving the other fields. Thus, there are non-calculable contributions to the masses-squared that scale as $^{3}$

$$
m_{\text {non-calc }}^{2} \sim f_{B}^{2} / \Lambda^{2} \sim r_{\mathrm{UV}}^{4 N-6} \Lambda^{2} .
$$

This contribution is small compared to the tree-level masses of all the fields in the model. The pseudo-modulus $B$, whose calculable mass only arises from the Coleman-Weinberg potential, requires a more detailed estimate. The radiatively generated $B$ mass depends on the relative sizes of $f_{B}$ and $m_{1} m_{2}$, where $m_{1}, m_{2}$ are the masses defined in Eq. (16). If $m_{1} m_{2}>f_{B}$ we have

$$
m_{B}^{2(C W)} \sim \frac{1}{16 \pi^{2}} f_{B}
$$

so that

$$
\frac{m_{\text {non-calc }}^{2}}{m_{B}^{2}(C W)} \sim 16 \pi^{2} \frac{f_{B}}{\Lambda^{2}}
$$

which is clearly small.

If however, as is the case in our model, $m_{1} m_{2}<f_{B}$, we have

$$
m_{B}^{2}{ }^{(C W)}=\frac{1}{16 \pi^{2}} \frac{m_{1}^{2} m_{2}^{2}}{f_{B}},
$$

so that

$$
\frac{m_{n o n-c a l c}^{2}}{m_{B}^{2(C W)}} \sim 16 \pi^{2} \frac{f_{B}^{3}}{\Lambda^{2} m_{1}^{2} m_{2}^{2}}
$$

\footnotetext{
3 The corresponding non-calculable contribution to the fundamental singlet $S_{I}$ additionally involves at least two powers of the corresponding singlet coupling $\lambda_{I}$.
} 
and whether or not this is smaller than one depends on the ratio $f_{B} /\left(m_{1} m_{2}\right)$. Using the parametric dependences of the various scales on $r_{\mathrm{UV}}$ of Eq. (6) we find

$$
\frac{m_{n o n-c a l c}^{2}}{m_{B}^{2}(C W)} \sim 16 \pi^{2} r_{\mathrm{UV}}^{2 N-5},
$$

so that for small $r_{\mathrm{UV}}$ the non-calculable contribution is subdominant in this case as well.

We can also see now why we chose the highest-dimension gauge invariant for the supersymmetry breaking tadpole. The O'Raifeartaigh model with

$$
W=\phi\left(\phi_{1} \phi_{2}+f\right)+m_{1} S_{1} \phi_{1}+m_{2} S_{2} \phi_{2} e
$$

has a minimum with $\phi_{1,2}=0$ for $f<m_{1} m_{2}$. Thus if we can achieve $f<m_{1} m_{2}$, the minimum is at the origin and the pseudo-modulus $\phi$ mass is given by Eq. (21), so that noncalculable contributions can indeed be neglected. In our model, however, the fields $\phi, \phi_{1,2}$

must be chosen among $B, \tilde{A}, X$, and $Y_{i j}$, which always results (for $\lambda_{I} \sim 1$ ) in $f_{\phi}>m_{1} m_{2}$. The $\phi_{1,2}$ VEVs are then nonzero, and in order for them to be small, one must choose $\phi$ to be the highest dimension field.

\section{Supersymmetric minima?}

One can still ask whether, in addition to the supersymmetry-breaking minimum near the origin, there are any supersymmetric minima or runaway directions elsewhere on the moduli space. As we saw above, supersymmetry can not be restored for finite field VEVs. However, the analysis performed so far does not rule out the possibility of runaway directions with supersymmetric solutions at infinity, because the Kähler potential written in terms of the gauge invariants can become singular at the boundary of field space. In particular, the conclusion that supersymmetry is broken hinges on the assumption that the correct weakly coupled degree of freedom is the baryon, which appears linearly, as opposed to the antiquark $\bar{Q}$. Thus for large $\bar{Q}$ VEVs, this conclusion might not hold. However, as saw in section IIB, the baryon flat direction is lifted. A nonzero $\bar{Q} \mathrm{VEV}$ is only possible classically if either $S_{M}$ or $S_{P}$ go to infinity. To study the behavior of the theory at large field VEVs, we will now examine the dynamics along the classical flat directions. There are three types of singlet flat directions:

- $S_{M}$ gives mass to the vector-like flavors and may play a role in the cancellation of the baryon $F$-term.

- $S_{P}$ gives rise to a renormalizable superpotential coupling $A \bar{Q}^{2}$ in the low-energy theory and may also play a role in the cancellation of the baryon $F$-term

- $S_{I \neq M, P}$ give rise to NR superpotential couplings in the low-energy theory.

Let us now examine these directions in the quantum theory, starting with $S_{M} \rightarrow \infty$. Consider the $\bar{Q} F$-term equation:

$$
\lambda_{M} S_{M}^{i a} Q_{i}+\frac{\lambda_{P}}{M_{\mathrm{UV}}} S_{P}^{a b} A \bar{Q}_{b}+\frac{\lambda_{B}}{M_{\mathrm{UV}}^{2 N-3}} \bar{Q}^{2 N-1}=0 .
$$


The classical potential can be made arbitrarily small if the flat direction $S_{M} \neq 0$ is approached at infinity $\mathrm{as}^{4}$

$$
S_{M} \rightarrow \infty, \quad B=\bar{Q}^{2 N-1} \neq 0, \quad Q \sim \bar{Q}^{2 N-1} / S_{M} \rightarrow 0 .
$$

While in this ansatz $\bar{Q}$ is arbitrary, it is constrained to be parametrically smaller than $S_{M}$. Thus at energies above $\bar{Q}$ and below $S_{M}$, four of the $\mathrm{SU}(2 N)$ flavors can be integrated out, and the resulting effective theory does not have baryons. This low-energy theory is the zero-flavor $\mathrm{SU}(2 N)$ with an antisymmetric tensor and $2 N-4$ anti-fundamentals coupled to some singlets. The dynamical superpotential of the effective theory depends on a power of $S_{M}$ with a specific exponent. In s-confining theories this exponent is always greater than 1 , so that the potential is a monotonically increasing function of $S_{M}$. Thus the $S_{M}$ flat direction is lifted non-perturbatively.

In contrast, the $S_{P}$ flat direction leads to a runaway in the quantum theory. To see this, note that it also can be approached at non-vanishing baryon VEV as ${ }^{5}$

$$
B=\bar{Q}^{2 N-1} \neq 0, \quad A \sim \bar{Q}^{2 N-2} / S_{P} \rightarrow 0 .
$$

However, unlike in the previous case, the $S_{P}$ VEV only generates a renormalizable Yukawa coupling $A \bar{Q}^{2}$ of order $\left\langle S_{P}\right\rangle / M_{\mathrm{UV}}$ in the effective theory, while the light degrees of freedom of this effective theory remain unchanged. As a result, the baryon direction still exists in the field space of the low energy theory. Studying the theory in the regime

$$
\Lambda \ll \bar{Q} \ll S_{P} \ll M_{\mathrm{UV}}
$$

both the gauge and the Yukawa interactions are weak, and the potential is calculable in terms of the elementary degrees of freedom. In particular, the potential along (29) can be made arbitrarily small and there is a runaway towards a supersymmetric vacuum at infinity (beyond the range of validity of the theory). Still, the supersymmetry-breaking minimum we found earlier is not destabilized since near the origin the Coleman-Weinberg potential generates a positive mass for $B$, while $S_{P}$ is massive due to the non-perturbative dynamics. We therefore have a local supersymmetry-breaking minimum at the origin, and a runaway direction towards zero potential for $B \gg \Lambda, S_{P} \rightarrow \infty$, with the two regions separated by a non-calculable potential barrier.

Finally, we do not expect any dramatic effect on the dynamics along the directions where only the $S_{I \neq M, P} \mathrm{VEVs}$ are turned on. An $S_{I \neq M, P} \mathrm{VEV}$ below $M_{\mathrm{UV}}$ simply gives rise to an irrelevant superpotential coupling, such as, for $S_{A}, A^{N}$. The analysis of section IIB remains valid in the regime of validity of the effective theory, $S_{I}<M_{\mathrm{UV}}$.

\section{E. An $R$-breaking example}

The IR theory described above preserves an $R$ symmetry which remains unbroken in the supersymmetry-breaking vacuum. This follows from the fact that this theory is an O'Raifeartaigh model with all $R$ charges being 0 or 2 [14], and with only a single modulus

\footnotetext{
4 This requires turning on additional singlet VEVs for the $F$-term equations to be satisfied.

${ }^{5}$ In this case too, other VEVs must be turned on for the remaining $F$-term equations to be satisfied.
} 


\begin{tabular}{|c|c|c|c|c|c|c|c|}
\hline & $S U(2 N+1)$ & $S U(2 N+1)$ & $S U(4)$ & $U(1)^{\prime}$ & $U(1)$ & $U(1)_{R}$ & $\operatorname{dim}$ \\
\hline$A$ & 日 & 1 & 1 & 0 & 4 & 0 & 1 \\
\hline $\bar{Q}$ & $\bar{\square}$ & $\square$ & 1 & 4 & 0 & $2 /(2 N+1)$ & 1 \\
\hline$Q$ & $\square$ & 1 & $\square$ & $-2 N-1$ & $-2 N+1$ & 0 & 1 \\
\hline$M_{i a} \sim\left(Q_{i} \bar{Q}_{a}\right)$ & & $\square$ & $\square$ & $3-2 N$ & $-2 N+1$ & $2 /(2 N+1)$ & 2 \\
\hline$P_{a b} \sim\left(A \bar{Q}^{2}\right)$ & & 日 & 1 & 8 & 4 & $4 /(2 N+1)$ & 3 \\
\hline$Y_{i} \sim\left(A^{N} Q\right)$ & & 1 & $\square$ & $-2 N-1$ & $2 N+1$ & 0 & $N+1$ \\
\hline $\bar{Y}^{i} \sim\left(A^{N-1} Q^{3}\right)$ & & 1 & $\bar{\square}$ & $-6 N-3$ & $-2 N-1$ & 0 & $N+2$ \\
\hline$B \sim(\bar{Q})^{2 N+1}$ & & 1 & 1 & $8 N+4$ & 0 & 2 & $2 N+1$ \\
\hline$S_{M}^{i a}$ & & $\bar{\square}$ & $\bar{\square}$ & $-3+2 N$ & $2 N-1$ & $2-2 /(2 N+1)$ & 1 \\
\hline$S_{P}^{a b}$ & & 日 & 1 & -8 & -4 & $2-4 /(2 N+1)$ & 1 \\
\hline$S_{Y}^{i}$ & & 1 & $\bar{\square}$ & $2 N+1$ & $-2 N-1$ & 2 & 1 \\
\hline$S_{\bar{Y} i}$ & & 1 & $\bar{\square}$ & $6 N+3$ & 0 & 2 & 1 \\
\hline
\end{tabular}

TABLE II: Matter content of the $S U(2 N+1)$ model. The quantum numbers for charged elementary fields are given in the top part, for gauge invariant composites in the middle part, and for gauge singlets in the bottom part.

of charge $2[9,16]$. One can easily modify the model in order to obtain spontaneous $R$ symmetry breaking, following [14]. As an example, starting from the superpotential Eq. (44), we can set $\lambda_{A}=0$, and add the term ${ }^{6}$

$$
\delta W_{0}=\frac{\lambda_{A s q}}{M_{\mathrm{UV}}^{2 N-3}}\left(A^{N}\right)^{2}
$$

which becomes a quadratic term in $\tilde{A}$ in the IR. Intergrating out $M, P$ and the corresponding singlets as before, as well as $Y$ and $S_{Y}$, one is left with the superpotential

$$
W=B\left(\tilde{A} X+f_{B}\right)+m_{X} S_{X} X+m_{A s q} \tilde{A}^{2},
$$

where $m_{A s q}=\lambda_{A s q} r_{\mathrm{UV}}^{2 N-3} \Lambda$, which reproduces the simplest R-breaking model of [14]. As discussed in [14], this model has a runaway direction along $S_{X}$, but for $f_{B}<m_{X} m_{A s q}$, one can get a local stable minimum near $B=0$. This condition requires some tuning of the parameters. Specifically, we need to take $\lambda_{B}<r_{\mathrm{UV}}^{N}$.

\section{THE ODD- $n$ SINGLE ANTI-SYMMETRIC TENSOR SU( $n)$ THEORIES}

It is straightforward to repeat the above analysis for the s-confining $\mathrm{SU}(2 N+1)$ theories with a single anti-symmetric tensor. Again, these theories have 4 "extra" flavors. Their matter content and symmetries are given in Table II] As we will see, the construction of a supersymmetry breaking IR model is even simpler in this case. Coupling singlet fields to all

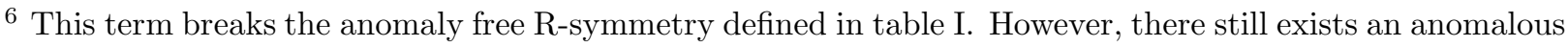
R-symmetry under which the tree-level superpotential is invariant. As shown in [17], this anomalous Rsymmetry becomes an accidental R-symmetry of the IR description. 
the gauge invariants apart from $B$ and writing down the full superpotential,

$$
\begin{aligned}
W & =\left[Y M^{3} P^{N-1}+\bar{Y} M P^{N}+m_{M} S_{M}^{i a} M_{i a}+m_{P} S_{P}^{a b} P_{a b}\right] \\
& +\left[B\left(\bar{Y} Y+f_{B}\right)+m_{Y} S_{Y} Y+m_{\bar{Y}} S_{\bar{Y}} \bar{Y}\right]
\end{aligned}
$$

we see that once again the superpotential factorizes into two parts, with $M$ and $P$ appearing only in the NR terms of the dynamical superpotential. The remaining fields give rise to an O'Raifeartaigh model in the IR. There is a calculable, supersymmetry breaking vacuum near the origin. As in the even- $n$ case, with no tuning of order one couplings we have $f_{B}>m_{Y} m_{\bar{Y}}$, and at the minimum all VEVs are zero apart from

$$
Y \sim r_{\mathrm{UV}}^{N-1 / 2} \Lambda, \quad \bar{Y} \sim r_{\mathrm{UV}}^{N-3 / 2} \Lambda
$$

Just as for the even- $n$ case, the $S_{M}$ direction is lifted quantum mechanically, but there is a runaway direction along $S_{P} \neq 0$.

\section{OTHER GENERALIZATIONS}

We can generalize our construction to almost all the s-confining theories. Here we will briefly study a few examples.

Consider $S U(N)$ SQCD with $F=N+1$ flavors [5]. The gauge invariants of the theory are the mesons $M_{I a}=Q_{I} \bar{Q}_{a}$, the baryons $B^{I}=\left(Q^{N}\right)^{I}$, and the anti-baryons $\bar{B}^{a}=\left(\bar{Q}^{N}\right)^{a}$, with $I, a=1, \ldots, F$. We add singlets for all of these gauge invariants apart from $B^{F}$. We also add the superpotential ${ }^{7}$,

$$
W_{\text {class }}=\lambda_{S} S_{I a} M_{I a}+\frac{\bar{\lambda}_{X}}{M_{\mathrm{UV}}^{N-2}} \bar{X}_{a} \bar{B}^{a}+\frac{\lambda_{X}}{M_{\mathrm{UV}}^{N-2}} X_{i} B^{i}+\frac{\lambda_{B}}{M_{\mathrm{UV}}^{N-3}} B^{F} .
$$

where $i=1, \ldots, N$, and we wrote the superpotential in terms of invariants for brevity. Once again, the only classical flat directions preserved by this superpotential are parameterized by the gauge singlets $X, \bar{X}$ and $S$.

The IR superpotential, written in terms of canonically normalized fields, is given by

$$
W=B^{I} M_{I a} \bar{B}^{a}+m_{M} S_{F a} M_{F a}+\bar{m}_{B} \bar{X}_{a} \bar{B}^{a}+m_{M} S_{i a} M_{i a}+m_{B} X_{i} B^{i}+B^{F} f_{B},
$$

with $f_{B} \sim \lambda_{B} r_{\mathrm{UV}}^{N-3}, m_{M} \sim \lambda_{S} \Lambda, \bar{m}_{B} \sim \bar{\lambda}_{X} r_{\mathrm{UV}}^{N-2} \Lambda$, and $m_{B} \sim \lambda_{X} r_{\mathrm{UV}}^{N-2}$. Here we omitted the non-renomalizable $\operatorname{det} M$ term. Near the origin, the non-perturbative dynamics marries the singlets with the gauge-invariant composites. The mesons $M_{i a}$ and their singlets $S^{i a}$ get mass of order $\Lambda$, while $X_{i}, B^{i}$ get mass $\sim r_{\mathrm{UV}}^{N-2} \Lambda$. Since the mesons $M_{i a}$ are stabilized near the origin, the $\operatorname{det} M$ term in the dynamical superpotential is irrelevant near the minimum. In the IR one then has an O'Raifeartaigh model and the Coleman-Wienberg potential stabilizes $B^{F}$ at the origin. Note that in this case, we need to take some couplings in (35) to be small in order to get a calculable minimum. With all couplings of order $1, f_{B}>m_{M} \bar{m}_{B}$ and the

\footnotetext{
${ }^{7}$ A slightly different deformation of s-confining SQCD was considered in [17]. There the superpotential was designed to lead to R-symmetry breaking. On the other hand, our goal here is to illustrate the result of lifting all but one gauge invariant moduli through couplings to singlets.
} 


\begin{tabular}{|c|c|c|c|c|c|c|}
\hline & $S U(5)$ & $S U(3)$ & $S U(3)$ & $U(1)$ & $U(1)_{R}$ & $\operatorname{dim}$ \\
\hline$A$ & $\boxminus$ & $\square$ & 1 & 1 & 0 & 1 \\
$\bar{Q}$ & $\bar{\square}$ & 1 & $\square$ & -3 & $2 / 3$ & 1 \\
\hline \hline$X=A \bar{Q}^{2}$ & 1 & $\square$ & $\square$ & -5 & $-4 / 3$ & 3 \\
$Y=A^{3} \bar{Q}$ & 1 & $\square$ & $\square$ & 0 & $2 / 3$ & 4 \\
$Z=A^{5}$ & 1 & $\square$ & 1 & 5 & 0 & 5 \\
\hline
\end{tabular}

TABLE III: Matter content of the $S U(5)$ model.

$\bar{B}$ VEVs turn out to be large. Choosing $\lambda_{B} /\left(\lambda_{S} \bar{\lambda}_{X}\right)<r_{\mathrm{UV}}$, we have instead $f_{B}<m_{M} \bar{m}_{B}$, so that all the fields are stabilized at the origin.

One can check that in this case, all the flat directions are lifted quantum mechanically.

Another easy to analyze example is the $S U(5)$ model with three "generations" of an antisymmetric tensor and one anti-fundamental. The matter content of the model is presented in Table III. It was shown in [10] that the tree-level superpotential given by the sum of all the gauge invariant moduli leads to ISS type metastable SUSY breaking. We will instead add singlets so that the full superpotential is

$$
W=\frac{1}{\Lambda^{12}}\left(X Y Z+Y^{3}\right)+S_{Y} Y+S_{Z} Z+X
$$

giving an O'Raifeartaigh model in the IR with a supersymmetry breaking minimum near the origin. Again, there are runaway directions along $S_{Y} \neq 0$ and $S_{Z} \neq 0$ for large $A$ VEVs.

One can similarly repeat this construction for theories 3.1 .6 or 3.1 .9 of [8] ]

\section{CONCLUSIONS}

We presented a simple recipe for obtaining effective O'Raifeartaigh models in the IR from s-confining theories coupled to singlets. This construction results in calculable, local supersymmetry-breaking minima near the origin in many of the s-confining $\mathrm{SU}(N)$ theories.

It is important to note that our construction relies on the presence of one or more dynamically-generated terms that are marginal in the infrared. Thus not all s-confining theories can lead to SUSY breaking in a non-trivial way. For example, the $S U(7)$ theory with two fields in the symmetric- and six fields in the anti-symmetric representation has two moduli, and its dynamical superpotential is quartic in the moduli [8]. Thus it does not give rise to an O'Raifeartaigh model in the IR. Indeed the superpotential

$$
W=\frac{1}{\Lambda^{13}} N^{2} H^{2}+N+S H
$$

where, following [8], $H$ and $N$ denote the $S U(7)$ composites while $S$ is the gauge singlet, has a runaway direction along which $H \rightarrow 0$ while $S, N \rightarrow \infty$.

The recipe we described can be easily applied to the $\mathrm{SO}$ and $\mathrm{SP}$ s-confining theories. It would be interesting to generalize it to theories with weakly coupled IR duals as well. 


\section{ACKNOWLEGMENTS}

The research of Y. Shadmi was supported by the Israel Science Foundation (ISF) under grant No. 1155/07, and by the United States-Israel Binational Science Foundation (BSF) under grant No. 2006071. Y. Shirman was supported by the National Science Foundation under grant PHY-0970173.

[1] E. Witten, Phys. Lett. B105, 267 (1981).

[2] See for example: E. Poppitz, S. P. Trivedi, Ann. Rev. Nucl. Part. Sci. 48, 307-350 (1998). hep-th/9803107]; Y. Shadmi, Y. Shirman, Rev. Mod. Phys. 72, 25-64 (2000). hep-th/9907225]; K. A. Intriligator, N. Seiberg, Class. Quant. Grav. 24, S741-S772 (2007). hep-ph/0702069; M. Dine, J. D. Mason, Rept. Prog. Phys. 74, 056201 (2011). arXiv:1012.2836 [hep-th]].

[3] K. A. Intriligator, N. Seiberg, D. Shih, JHEP 0604, 021 (2006). hep-th/0602239.

[4] See for example: G. F. Giudice, R. Rattazzi, Phys. Rept. 322, 419-499 (1999). hep-ph/9801271.

[5] N. Seiberg, Phys. Rev. D49, 6857-6863 (1994). hep-th/9402044.

[6] K. A. Intriligator, P. Pouliot, Phys. Lett. B353, 471-476 (1995). hep-th/9505006.

[7] P. Pouliot, Phys. Lett. B367, 151-156 (1996). hep-th/9510148.

[8] C. Csaki, M. Schmaltz, W. Skiba, Phys. Rev. D55, 7840-7858 (1997). hep-th/9612207.

[9] Y. Shadmi, JHEP 1108, 149 (2011). arXiv:1107.3565 [hep-th]].

[10] T. Lin, J. D. Mason, A. Sajjad, JHEP 1108, 145 (2011). arXiv:1107.2658 [hep-th]].

[11] K. A. Intriligator, S. D. Thomas, Nucl. Phys. B473, 121-142 (1996). hep-th/9603158.

[12] K. -I. Izawa, T. Yanagida, Prog. Theor. Phys. 95, 829-830 (1996). hep-th/9602180.

[13] V. P. Spiridonov, G. S. Vartanov, Commun. Math. Phys. 304, 797-874 (2011). arXiv:0910.5944 [hep-th]].

[14] D. Shih, JHEP 0802, 091 (2008). hep-th/0703196 [HEP-TH]].

[15] Z. Chacko, M. A. Luty, E. Ponton, JHEP 9812, 016 (1998). [hep-th/9810253].

[16] J. L. Evans, M. Ibe, M. Sudano, T. T. Yanagida, arXiv:1103.4549 [hep-ph]].

[17] J. Goodman, M. Ibe, Y. Shirman, F. Yu, Phys. Rev. D84, 045015 (2011). arXiv:1106.1168 [hep-th]]. 